\title{
Interior Environmental Design, Heal?
}

\author{
Naglaa Sami Abdelaziz* \\ Department of Interior Design, Ajman University, United Arab Emirates

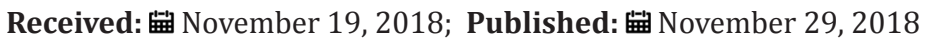 \\ *Corresponding author: Naglaa Sami Abdelaziz, Interior Design, Ajman University, United Arab Emirates
}

\section{Introduction}

Alternative medicine, from the first though, relate our minds to herbs that are the substitution of the chemicals treatments, in fact, they are the origin of these chemicals. Actually, the alternative healing process has several paths; the oral treatments (herbs and nutrition), the external or applicable treatments (oils and natural products, and acupressure points), soundscape (music and rhythms), scentscape (the aromatic herbs), and finally the least eminent is the perception of the surrounds (the interior design and its components from materials textures, colors, lines, shapes, and forms). The interior environment design, which still not famous as an alternative healing option, explore various elements that affect immensely the human' healing. Paper and research discussed this point are few. However, the healing by color starts to be prominent. This article shall explore the elements of interior environmental design that does have great effects on the human' healing as an alternative and complementary medicine.

\section{Discussion}

Interior is where humans live, work, and get entertainment. It is our surround either in an enclosed or open place. These spaces do have a physical layout that fit the need functions. However, the interior design elements do affect consciously then unconsciously each person depend on each' background, education, personality, and culture. The interior design has vast scope, and many academics and researchers deal with it from the physical aspect. This profession exceeds the solidity and the materiality selections to the mental aspects, from the conscious perception of the unconscious acuity. Each of its elements after their perception, they affect the human at different levels and ways. Emotional and cognitive responses play major roles in the interior environmental design. Awake or asleep we all listen, touch, and smell. While awake, we all see, hear, taste, touch \& smell. Each image, sound, taste, substantial \& scent send sensory information to our brain, where we process \& respond accordingly! Responses are emotional or/and cognitive. The person culture, education, and background effects on the cognitive responses. The cognition relies on conscious perception wherever the person uses his/her surrounds. While the cognition perception and emotional senses effects on the overall emotional responses. Each person in space "processes" the available information he/she perceive unconsciously (automatically, involuntary). Emotional responses reflect our dominant sense (based on culture and personality). When exploring the interior environmental design elements, that have psychological effects and thus could affect the human health, we can categorize them based on the human senses. Well, they all play a certain role in the interior environment, except the taste sense, and they are as following:

a) The visual sense is the most common as a design feature and the least common in the alternative healing process, except the color effects. Seeing, as vital and influencing sense perform a key part in our health. As much human see a safe surround, the overall physical conditions raise. First element perceived is the color. Many protruding studies used it today as an essential alternative healing process. Colors could raise or lower the environment temperature perception and consequently the body ones. Visual proportions, lines, and forms come in sequence to the color. The golden ratio or the proportion to perfection triggers the brain waves in pleasurable ways. Lines and forms indorse the visual quality of the interior and consequently the positives ambiances that play enormously as alternative solutions for healing. Each type of line and form lead to a certain psychological human reaction that thrills the human health.

b) The hearing sense, as it is the sense that does not stop by sound scape, but surpasses to the heart rate. It depends on the required achievements; the rhythm could adjust them. As faster the rhythm as faster the heart rate and vice versa. Just by the surrounding soundscape, we can control our physical health, especially the heart rates. It goes the same for the type of sound from simple tones to the complex ones, from the natural ones to the human-made ones; soundscape plays a major role as an alternative healing substitute. Contrary, noise, with extreme level, can lead to direct death!

c) The sense of touching, consciously or unconsciously, affects our momentary needs depend on the messaging we perceive. Touching creates such message that leads to different sensations and thus creates modified health issues related to the type of texture surrounded, smooth or rough. 
d) The smell sense is our most idiosyncratic, particular, and basic sense. The scent is an additional element to create the psychological effect. After a few minutes of smelling, it affects unconsciously. It has an effective part in the overall human functionality. Scents play an effective role in mental and physical tasks; also, it enhances relaxation, memories, and creativity. As much the scents could improve sleeping, reduces anxiety and tension besides the mood improvement and increasing the alertness. The overall health improve by scents. Each of these conditions refers to a specific type of scent, herbal as essential treatment tool. The scent affects the overall health in term of condition and quantity of changes required.

\section{Conclusion}

Interior environmental design is the interior design that deals with the psychological effects of its components and creates, based on, the required reaction. Each of the five senses, except the taste, could be a portal to an additional healing treatment "alternative" to the traditional or common healing procedure. The studies need further explorations and experiments to reach protruding results in favor of humanity. It is a collaboration between the interior designer and the psychologist and the physiologist to rescue the human being from all these chemicals that the entire physician confirms their side effects, yet the positive reaction could not be negligee.

As all the interior environmental elements that create a supplementary state to the general health, the ethnic group as well as the gender play additional role to the culture and the educational backgrounds of the users. Each of these senses participates, to some extent, in the physical and mental human' health. They could be the major alternative healing in the next future since all humanity is revolving now on the green, organic, and sustainable living.

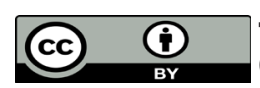

This work is licensed under Creative Commons Attribution 4.0 License

To Submit Your Article Click Here:

Submit Article

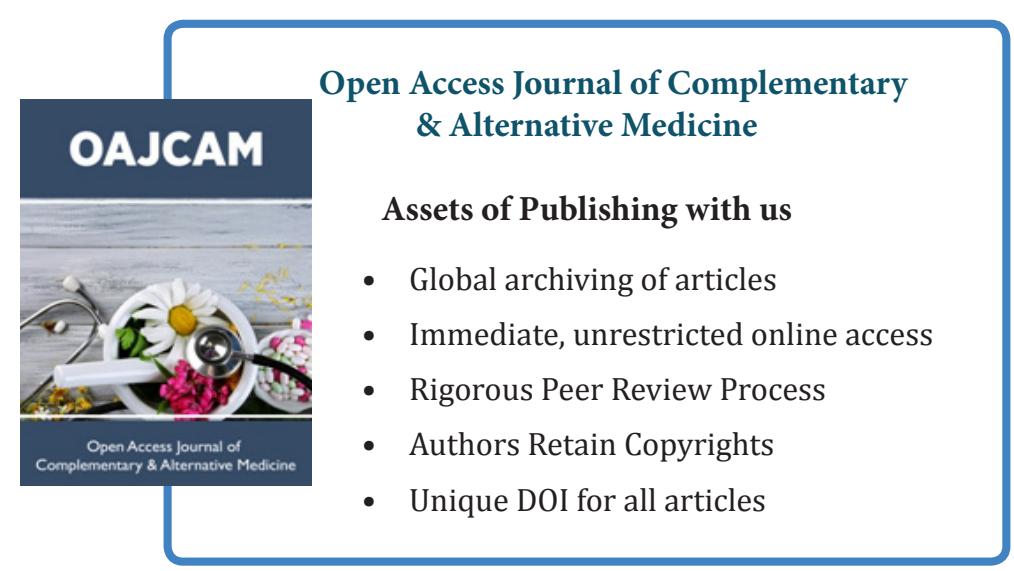

